\title{
Splenic artery aneurysm in Sjögren's syndrome
}

\author{
Cuneyt Tetikkurt, Zeynep Ferhan Ozseker, Fatma Gülsüm Karakaş \\ Pulmonary Diseases Department, Cerrahpasa Medical Faculty, Istanbul Cerrahpasa University, Turkey
}

\begin{abstract}
A 40-year-old female presented with cough, exertional dyspnea, abdominal pain with distention, fatigue, dry eyes and dry mouth. Past history revealed asthma. Physical examination was normal except for tachypnea. We found leukocytosis, azygos fissure on chest X-ray along with normal pulmonary function tests and arterial blood gases. Thorax computed tomography (CT) revealed bronchiectasis and ground glass opacities in both lungs. Abdominal CT demonstrated thrombosed proximal splenic artery aneurysm. Further diagnostic procedures were done and according to the positive Schirmer test and compatible histopathologic findings of the salivary gland, diagnosis of primary Sjögren's syndrome was established. Splenic artery aneurysm is rare occurring in less than $1 \%$ of the population that usually appears as an incidental finding. This is the first case in literature that introduces Sjögren's syndrome as a risk factor for splenic artery aneurysm. The silent presentation of the splenic artery aneurysm should previse the clinicians that such an occurrence may cause a significant diagnostic dilemma.
\end{abstract}

Correspondence: Cuneyt Tetikkurt, Pulmonary Diseases Department, Cerrahpasa Medical Faculty, Istanbul Cerrahpasa University, Tanzimat sokak Serkan Apartman1, No 8/16, Caddebostan 34728, Istanbul,Turkey.

Tel. +90.532 .3810900 - Fax: +90.212 .5870217 .

E-mail: tetikkurt@gmail.com

Contributions: CT, manuscript design and writing; ZFO, patient's follow-up, laboratory data analysis; GK, literature searching. All authors approved the final version to be published.

Conflict of interest: The Authors declare no conflict of interest.

Key words: Sjögren's syndrome; splenic artery; aneurysm.

Received for publication: 17 April 2019.

Accepted for publication: 20 June 2019.

CCopyright C. Tetikkurt et al., 2019

Licensee PAGEPress, Italy

Monaldi Archives for Chest Disease 2019; 89:1082

doi: 10.4081/monaldi.2019.1082

This article is distributed under the terms of the Creative Commons Attribution Noncommercial License (by-nc 4.0) which permits any noncommercial use, distribution, and reproduction in any medium, provided the original author(s) and source are credited.

\section{Introduction}

Aneurysm of the splenic artery is very rare while it is the most frequent site for visceral arterial aneurysms [1-3]. Rupture carries a substantial mortality risk and a severe outcome may come out as the initial manifestation [4,5]. Most of the splenic artery aneurysms are clinically silent and are incidentally diagnosed. Fatal complications may occur if the diagnosis is delayed. The clinical presentation and the laboratory findings were not suggestive for a splenic artery aneurysm diagnosis in our case. On the other hand, Sjögren's syndrome is a chronic autoimmune disease with exocrine gland dysfunction as a result of lymphocytic infiltration that may lead to arterial aneurysms. Although various arterial aneurysms have been described in Sjögren's syndrome, splenic artery aneurysm due to this syndrome has not been reported previously [6-10]. We present this patient as it is the first splenic artery aneurysm case in the literature associated with the Sjögren's syndrome. Second, its silent atypical profile may evoke a diagnostic impasse for the clinicians.

\section{Case report}

A 40-year-old female was admitted for cough, exertional dyspnea, abdominal pain with distension in the left upper quadrant, fatigue, dry eyes and a dry mouth for nine months. Dyspnea had deteriorated in the last six weeks. The patient was a non-smoker. Past history revealed asthma, one abortus, one curettage and two children. Her mother died of myocardial infarction while her father had diabetes mellitus, hypertension and stroke. Physical examination did not reveal any abnormal findings except for a tachypnea of 20 breaths per min. Her laboratory findings were within normal limits except leukocytosis and mild anemia with ERS: $30 \mathrm{~mm} / \mathrm{h}$, CRP: $3.7 \mathrm{mg} / \mathrm{lL}$, glucose: $99 \mathrm{mg} / \mathrm{dl}$, creatinine: $0.63 \mathrm{mg} / \mathrm{dl}$, urea: $39 \mathrm{mg} / \mathrm{dl}$, SGOT: $14 \mathrm{IU} / \mathrm{dl}$, SGPT: $17.7 \mathrm{IU} / \mathrm{dl}$ and LDH: $187 \mathrm{IU} / \mathrm{l}$. WBC was 16,200 cells $/ \mathrm{mm}^{3}$, $\mathrm{Hgb}: 11.1 \mathrm{~g} / \mathrm{dlL}$ and Htc: $34.1 \%$. Viral hepatitis screen and anti-HIV were negative. The electrocardiogram (ECG) showed sinus rhythm of $84 /$ min. Urine analysis was normal. Chest X-ray showed azygos fissure (Figure 1). Tuberculin test was $10 \mathrm{~mm}$. Pulmonary function tests revealed normal values with FVC: $1.82(73 \%), \mathrm{FEV}_{1}$ : 1.68 (79\%), $\mathrm{FEV}_{1} / \mathrm{FVC}$ : 93\%, PEF: 6.58 (116\%) and $\mathrm{MEF}_{25-75}$ : $4.12(122 \%)$. Arterial blood gas values revealed $\mathrm{pO}_{2}: 83.8, \mathrm{pCO}_{2}$ : $40.8 \mathrm{~mm} \mathrm{Hg}, \mathrm{HCO}_{3}: 25.9 \mathrm{mmol} / \mathrm{L}, \mathrm{pH}: 7.41, \mathrm{SaO}_{2}: 97.5 \%$ and were within normal limits. Thorax CT revealed ground glass opacities, bronchiectasis and fibrotic changes in both lungs (Figures 2 and 3) compatible with lung involvement of the Sjögren's syndrome. The diffusing capacity divided by the alveolar volume (DLCO/VA) was done because the patient had interstitial changes on the CT scan. DLCO/VA revealed a mildly decreased value of 
$3.40 \mathrm{ml} / \mathrm{mmHg} / \mathrm{min} / 1$ (predicted: 5.69; 60\%). Bronchoscopic examination was normal and BAL cytology revealed lymphocytic alveolitis. Immunology testing was done. Antinuclear antibody (ANA) and rheumatoid factor (RF) were positive while all other antibodies remained negative. RF was $24.4 \mathrm{IU} / \mathrm{ml}$ and ANA was $1 / 2560 \mathrm{IU} / \mathrm{ml}$ with a speckled pattern. Abdominal CT revealed a saccular aneurysm of the partially thrombosed $18 \mathrm{~mm}$ splenic artery (Figure 4), while abdominal ultrasound, CT and gastrointestinal endoscopy performed for pain with distention two years ago revealed normal findings. Schirmer test was positive with a 0 $\mathrm{mm}$ strip paper result that indicated xerophthalmia. Histopathologic examination of the salivary gland revealed extensive lymphoid infiltrates with germinal centers, limited interstitial fibrosis and acinar atrophy. Rheumatology consultation identified the Sjögren's syndrome in the patient. The final diagnosis was splenic artery aneurysm associated with Sjögren's syndrome. The patient is currently under conservative management with CT follow-up every six months.

\section{Discussion}

Sjögren's syndrome is a relatively common autoimmune disorder affecting $2-3 \%$ of the adult population. It is characterized by lymphocytic infiltration and destruction of the exocrine glands. The salivary and lacrimal glands are primarily affected, leading to a dry mouth and dry eyes, as the hallmark of the disease. Other exocrine glands, which may be affected, include those of the pancreas, bronchial tree and gastrointestinal tract. Sjögren's syndrome may occur as primary or in association with other connective tissue diseases as secondary. The spectrum of clinical manifestations of Sjögren's syndrome is wide ranging from mucosal dryness directly relevant to exocrine dysfunction to more systemic complaints affecting mainly the musculoskeletal, pulmonary, renal and vascular systems. Almost half of the highly selected Sjögren's syndrome patients with active CNS disease have small vessel vasculitis such as stenosis, aneurysm or occlusion of the small cerebral blood vessels [11-15]. Splenic artery aneurysm is the most common among visceral arterial aneurysms that constitute $60 \%$ of similar lesions [16]. Exact prevalence is unknown as most of these aneurysms have a silent clinical profile and are asymptomatic. Autopsy studies reveal a prevalence between $0.01 \%$ and $10.4 \%$ while they are identified coincidentally on $0.78 \%$ of the angiograms $[2,17,18]$. The asymptomatic clinical course and the lack of clinical suspicion may lead to a fatal outcome that may be as high as $70 \%$ to $90 \%$ due to the lethal consequences of a ruptured splenic artery aneurysm [19]. As arterial aneurysms of different locations are a known feature of Sjögren's syndrome, our case is unique for its presentation with a splenic artery aneurysm as the first patient in literature.

The only symptom of our patient was abdominal pain with distention that may be poorly attributed to the splenic artery aneurysm and easily go unnoticed while the final diagnosis was reached by performing almost an incidental abdominal CT due to this nonspecific manifestation. Dryness of the pharynx and esophagus due to lack of saliva along with impaired gastric emptying in primary Sjögren's syndrome may lead to deglutition, impaired clearance of acid, gastroesophageal reflux and esophagitis [20-21]. Previous studies have demonstrated that aortic aneurysms and cerebral artery pathologies are more prevalent in patients with autoimmune diseases compared with the general population, including rheumatoid arthritis, systemic lupus erythematosus and in Sjögren's syndrome. Several molecular mechanisms associated with the
Sjögren's syndrome may participate in the onset and progression of aortic aneurysms and dissection thereby suggesting that the Sjögren's syndrome is associated with aortic disorders including aortitis and aneurysm [22-24]. There are some case reports in the literature relevant to Sjögren's syndrome and aortic disease $[25,26]$. Clinical data from 10,941 patients and 43,764 control subjects extracted from Taiwan's National Health Insurance Research Database between 2000 and 2010 revealed that Sjögren's patients had significantly a higher prevalence of aortic aneurysm or dissection compared to controls $(0.43 \%$ vs. $0.37 \%)$ after a follow-up period of ten years. Further analysis also disclosed that Sjögren's

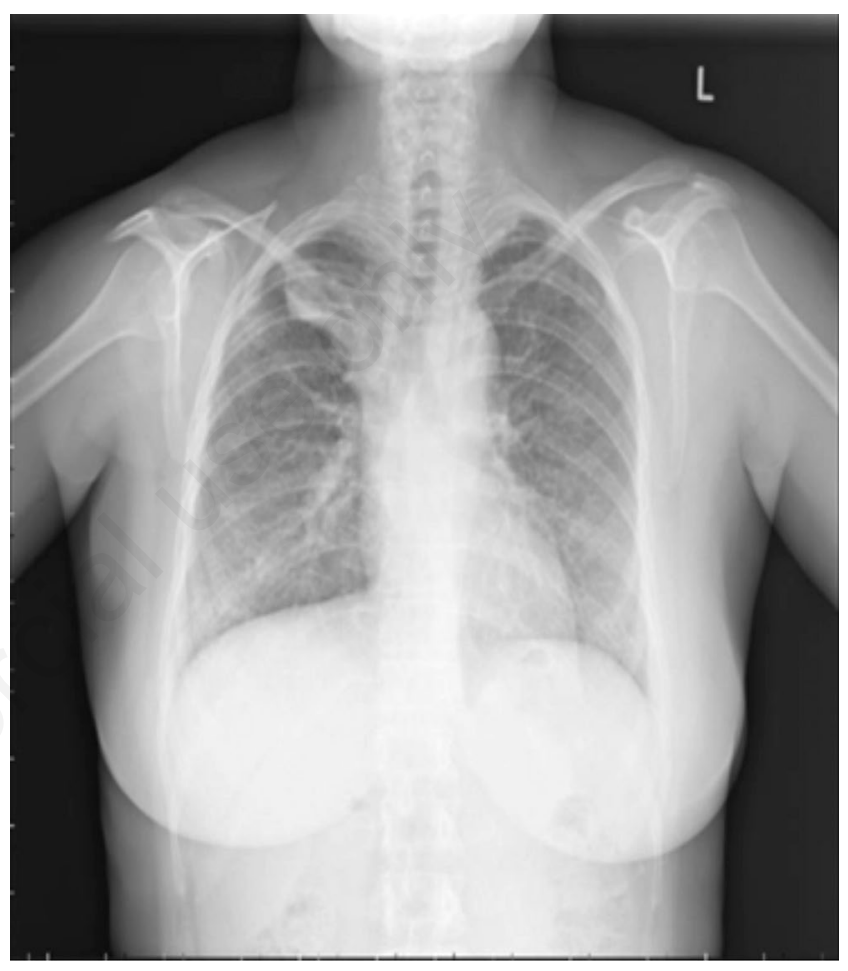

Figure 1. Normal chest X-ray showing azygos fissure.

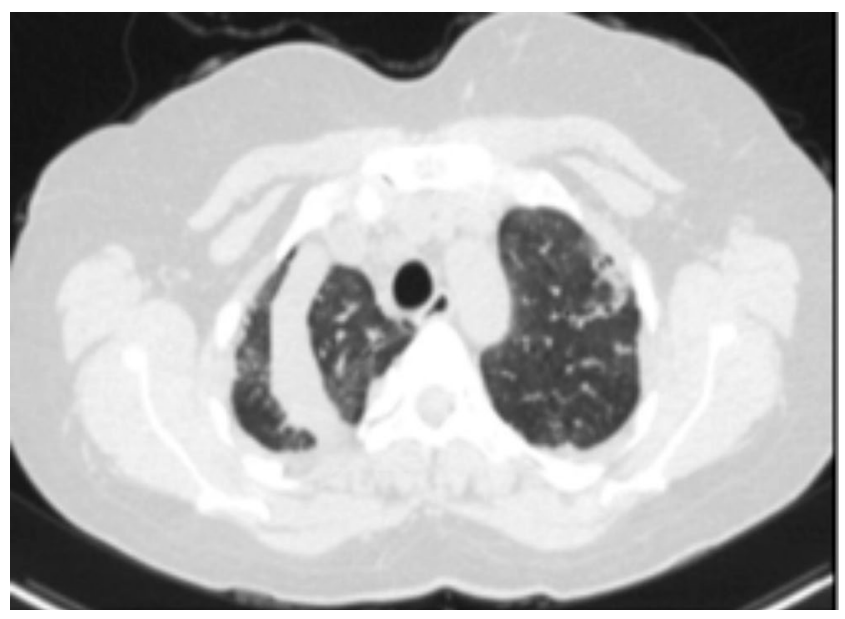

Figure 2. Chest CT revealing azygos fissure, fibrotic lesions and bronchiectasis. 
patients whether primary or secondary had a significantly higher risk of developing both aortic aneurysm and dissection in regard to control subjects [25]. These findings strongly suggest that Sjögren's patients have a higher risk of developing an aortic aneurysm and dissection. Although Sjögren's syndrome constitutes a risk factor for inflammatory artery disease relevant to aortic and cerebral arteries, there are no previous data in the literature reporting the presence of a splenic artery aneurysm in these patients. Our findings elicit that splenic artery aneurysm may occur in Sjögren's syndrome even if the clinical profile of the patient is silent or irrelevant that may lead to a fatal outcome if unidentified on time. The primary mechanism for the splenic artery aneurysm is probably the lymphocytic infiltration and the destruction of the vessel wall. The splenic artery aneurysm in this patient is due to the inflammatory arteritis that may be associated with the lymphocytic alveolitis of the lung both of which may have arisen from a common autoimmune pathway that has led to destructive lesions in various organs in the Sjögren's syndrome. Inflammatory lymphocytic interstitial inflammation has caused bronchiectasis along with fibrotic lesions



Figure 3. Chest CT showing bronchiectasis and ground glass opacities in both lungs. in the lung while the perivascular lymphocytic infiltration may have precipitated the splenic artery aneurysm.

\section{Conclusions}

Splenic artery aneurysms are extremely rare. Diagnosis requires a high index of clinical suspicion as most of the patients are asymptomatic, clinically silent or present with symptoms irrelevant to the splenic artery aneurysm. Due to the high rate of mortality following an aneurysmatic rupture, identification of a splenic artery aneurysm is highly crucial. Although many different locations for aneurysmal dilatation including the aortic and cerebral arteries have been reported in Sjögren's syndrome, our case is the first in literature that reveals a splenic artery aneurysm in these patients. The clinicians should bear in mind that Sjögren's syndrome may constitute a risk factor for splenic artery aneurysm and such an occurrence may arise in the presence of an unusually silent clinical profile that may lead to a fatal outcome. The primary mechanism for the splenic artery aneurysm in our patient is probably the lymphocytic infiltration of the perivascular wall due to an autoimmune expedient of Sjögren's syndrome that has induced similar lesions in the lung parenchyma consisting of bronchiectasis with fibrotic lesions as a result of lymphocytic alveolitis.

\section{References}

1. Ayalon A, Wiesner RH, Perkins JD, et al. Splenic artery aneurysms in liver transplant patients. Transplantation 1988;45:386-9.

2. Mattar SG, Lumsden AR. The management of splenic artery aneurysms: Experience with 23 cases. Am J Surg 1995; 169:580-4

3. Dave SP, Reis ED, Hossain A, et al. Splenic artery aneurysm in the 1990s. Ann Vasc Surg 2000;14:223-9.

4. De Vries JE, Schattenker ME, Malt RA. Complications of the
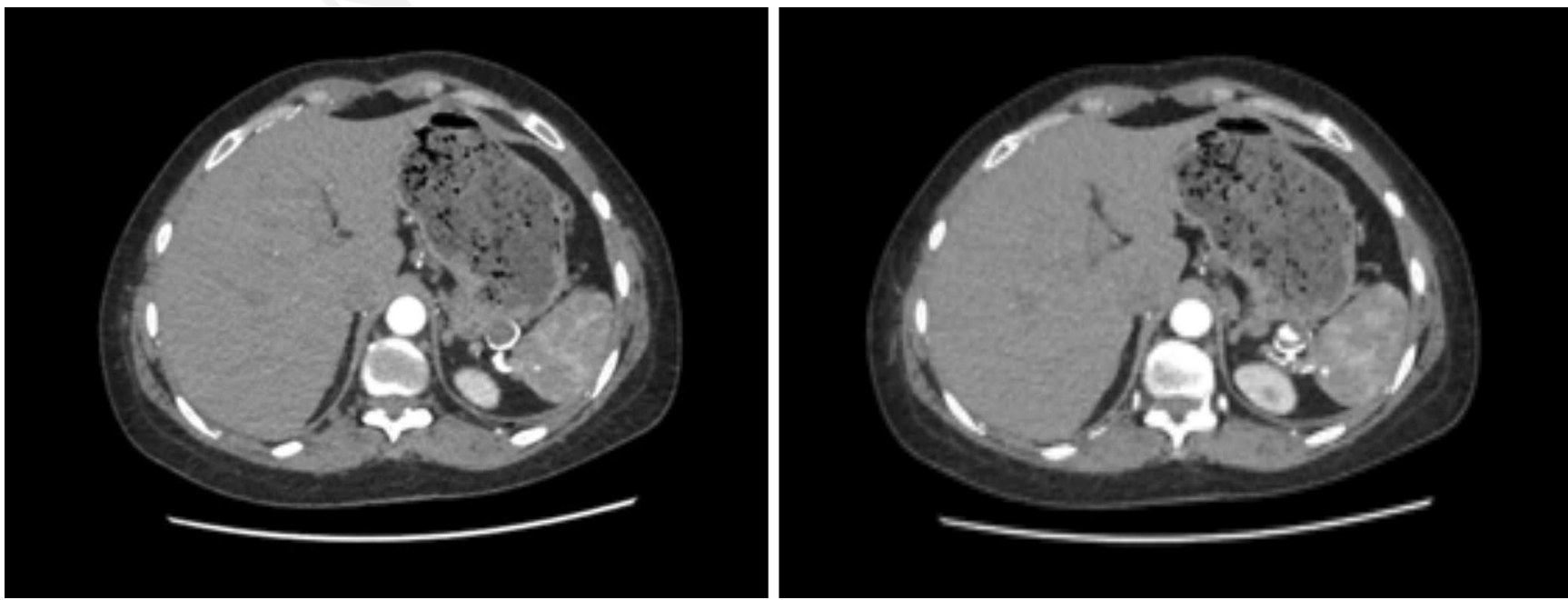

Figure 4. Abdominal CT revealing a partially thrombosed saccular $18 \mathrm{~mm}$ splenic artery aneurysm. 
splenic artery aneurysm other than intraperitoenal rupture. Surgery 1982;91:200-4.

5. Khurana J, Spinello IM. Splenic artery aneurysm rupture: a rare but fatal cause for peripartum collapse. J Intensive Care Med 2013;28:131-3.

6. Komatsu T, Mitsumura H, Yuki I, Iguchi Y. Primary Sjögren's syndrome presenting with multiple aneurysmal dilatation of cerebral arteries and causing repetitive intracranial hemorrhage. Neurol Sci 2016;365:124-5.

7. Tsai YD, Chien WC, Tsai SH, et al. Increased risk of aortic aneurysm and dissection in patients with Sjögren's syndrome: a nationwide population-based cohort study in Taiwan. BMJ 2018;8:e022326.

8. Ghinoi A, Pipitone N, Boiardi L, et al. Primary Sjögren's syndrome associated with chronic periaortitis. Rheumatology (Oxford) 2007;46:719-20.

9. Hayashi K, Morofuji Y, Suyama K, Nagata I. Recurrence of subarachnoid hemorrhage due to the rupture of cerebral aneurysms in a patient with Sjögren's syndrome. Case report. Neurol Med Chir 2010;50:658-61.

10. Haug ES, Skomsvoll F, Jacobsen G. Inflammatory aortic aneurysm is associated with increased incidence of autoimmune disease. J Vasc Surg 2003;38:492-7.

11. Lafitte C, Amoura Z, Cacoub P, et al. Neurological complications of primary Sjögren's syndrome. J Neurol 2001;248:577-84.

12. Alexander EL. Neurologic disease in Sjögren's syndrome: mononuclear inflammatory vasculopathy affecting central/peripheral nervous system and muscle. A clinical review and update of immunopathogenesis. Rheum Dis Clin North Am 1993;19:869-908.

13. Andonopoulos AP, Lagos G, Drosos AA, Moutsopoulos HM. The spectrum of neurological involvement in Sjögren's syndrome. Br J Rheumatol 1990;29:21-3.

14. Govoni M, Bajocchi G, Rizzo N, et al. Neurological involve- ment in primary Sjögren's syndrome: clinical and instrumental evaluation in a cohort of Italian patients. Clin Rheumatol 1999;18:299-303.

15. Tajima Y, Mito Y, Owada Y, et al. Neurological manifestations of primary Sjögren's syndrome in Japanese patients. Intern Med 1997;36:690-3.

16. Agrawal A, Whitehouse R, Johnson RW, Augustine T. Giant splenic artery aneurysm associated with arteriovenous malformation. J Vasc Surg 2006;44:1345-9.

17. de Perrot M, Bühler L, Deléaval J, et al. Management of true aneurysms of the splenic artery. Am J Surg 1998;175:466-8.

18. Wagner WH, Allins AD, Treiman RL, et al. Ruptured visceral artery aneurysms. Ann Vasc Surg 1997;11:342-7.

19. Fender GR, Haslett E, Leary T, et al. Management of splenic artery aneurysm rupture during trial of scar with epidural analgesia. Am J Obstet Gynecol 1999;180:1038-9.

20. Constantopoulos SH, Tsianos EV, Moutsopoulos HM. Pulmonary and gastrointestinal manifestations of Sjögren's syndrome. Rheum Dis Clin North Am 1992;18:617-35.

21. Hammar O, Ohlsson B, Wollmer P, Mandl T. Impaired gastric emptying in primary Sjogren's syndrome. J Rheumatol 2010;37:2313-8.

22. Shovman O, Tiosano S, Comaneshter D, et al. Aortic aneurysm associated with rheumatoid arthritis: a population-based crosssectional study. Clin Rheumatol 2016;35):2657-61.

23. Guy A, Tiosano S, Comaneshter D, et al. Aortic aneurysm association with SLE- a case-control study. Lupus 2016;25:959-63.

24. Robson JC, Kiran A, Maskell J ,et al. The relative risk of aortic aneurysm in patients with giant cell arteritis compared with the general population of the UK. Ann Rheum Dis 2015;74:129-35.

25. Lorenzo LR, Villegas AR, Mestre XM, et al. Aortic dissection after open repair of an infrarenal aortic aneurysm. Int J Angiol 2009; 18:143-6. 